modified AGREE and using systematic reviews to supplement the evidence base when there is discordance among recommendations.

\section{DEVELOPMENT OF MULTIDISCIPLINARY SYMPTOM MANAGEMENT TOOLS USING THE ADAPTE APPROACH}

I Harle, K Bak, S Molloy, T Green. Queen's University, Kingston, Canada, Cancer Care Ontario, Toronto, Canada

\section{0:1136/bmjqs-2013-002293.69}

Background Evidence-based tools for symptom management were developed to assist healthcare providers in comprehensive assessment and appropriate management of symptoms. These evidence-based tools consist of comprehensive guides-to-practice, quick reference pocket guides, algorithms and smart phone apps. Objectives The symptom management tools promote an interdisciplinary model of care that enables early identification and assessment of symptoms, appropriate documentation and communication regarding symptoms, optimal symptom management, and coordinated care throughout the illness trajectory. The tools are intended to be user-friendly, and are available in print, web and smart phone applications.

Methods The tools were developed by an interdisciplinary panel of healthcare providers using the ADAPTE guideline adaptation approach. This included a literature search for recent guidelines and systematic reviews, guideline appraisal using the AGREE tool and selection, and in some instances modification, of recommendations. Expert feedback was obtained and subsequently appropriate revisions were made.

Results The symptom management tools provide recommendations based on the best available evidence and expert consensus, for assessing, determining aetiology, diagnosing potential problems and for recommending non-pharmacological and pharmacological interventions.

Implications for Guideline Developers/Users The ADAPTE approach offers a comprehensive and rapid process of developing evidence-based tools for the cancer patient population. Following the global trend of creating user-friendly clinical guidance, the guides-to-practice, quick reference pocket guides, algorithms and smart phone apps are an innovative set of tools that are accessible to a diverse group of care providers, in a manner that would suit the individual's clinical needs.

\section{GUIDELINE DEVELOPERS' SELF-PERCEPTIONS OF ADHERENCE TO AND INTENTIONS TO ADHERE TO THE IOM STANDARDS}

1J Jue, 'L Haskell, 'S Cunningham, ${ }^{2} \mathrm{M}$ Nix, ${ }^{1} \mathrm{~V}$ Coates. ${ }^{1} E C R I$ Institute, Plymouth Meeting, USA; ${ }^{2}$ Agency for Healthcare Quality and Research, Rockville, USA

\section{0:1136/bmjqs-2013-002293.70}

Background The 2011 IOM report called for more rigorous and transparent development of guidelines. Compliance with the IOM Standards may be challenging for developers. Developer perception of their current adherence to the Standards gives insight into their understanding of them and the likelihood of adhering to them in the future.

Objectives (1) Assess developers' self-perceptions of adherence to the IOM standards (2) Assess developers' intentions to adhere to the IOM standards.
Methods This AHRQ funded work used a mixed-methods approach. We performed semi-structured telephone interviews and surveys to query developers about impressions of and intentions to implement the IOM standards in their CPGs. We also performed our own assessments of guidelines and compared them with developer self-ratings.

Results Of 14 developers, $43 \%$ utilised a systematic review to underpin their guidelines, and 57\% felt they would in the future. Funding sources were not disclosed by $46 \%$ of the developers. While $80 \%$ utilised an evidence rating scheme, fewer rated the recommendations. Notable differences between developer selfratings and researcher assessments of adherence occurred in several areas.

Discussion While some developers intend to improve processes to meet the Standards, others acknowledged they will not. Yet still others felt they already met the standards, but our assessment suggested a different estimation, revealing varying understanding among developers of the Standards.

Implications for Guideline Developers/Users The IOM standards will help identify rigorous and transparent evidence-based guidelines, but will pose implementation challenges. Education of developers on the Standards and expectations around them will be critical.

\section{CAN SYSTEMS FOR RATING EVIDENCE QUALITY AND RECOMMENDATION STRENGTH BE HARMONISED}

R Shiffman. Yale Center for Medical Informatics, New Haven, USA

\section{0:1136/bmjqs-2013-002293.71}

Background In 2011 the IOM recommended that every guideline recommendation should be accompanied by an indication of Evidence Quality and Recommendation Strength and called for strategies to encourage harmonisation of development processes. Objectives To characterise the differences in systems for rating Evidence Quality and Recommendation Strength as a prelude to possible standardisation.

Methods We examined 17 international, English-language guideline development systems to identify rating parameters and applied descriptive statistics. We also searched for conceptual linkages in the rating system descriptions and identified systems where Strength of Recommendation was stated as an Evidence Quality parameter.

Results Rating systems were remarkably inconsistent in their application of category indicators-using letters, Arabic and Roman numerals and combinations. The modal and median number of Evidence Quality categories was 3 (range 0 to 10) and Recommendation Strength categories was 4 (range 0 to 6). $13 / 17$ used randomised trials as indicators of highest quality evidence. 7 systems used "expert opinion," 6 used "case reports" or "case series," and 4 described "reasoning from first principles" to define lowest evidence quality. Definitions of intermediate levels varied considerably. 7 systems judged benefits and harms in deriving Recommendation Strength. In 7 rating systems, Strength of Recommendation was described entirely in terms of Evidence Quality.

Implication There is considerable disagreement about the requisite granularity and definition of categories of Evidence Quality and Recommendation Strength. Application of the concept of Recommendation Strength consonant with the IOM standard is limited. A straightforward mapping of rating systems to one another is elusive. 